\title{
ABNORMAL LEVELS OF INFLAMMATORY AND OXIDATIVE STRESS MARKERS IN PATIENTS WITH ARTERIAL HYPERTENSION AND DIABETES MELLITUS
}

\section{Maya P. Danovska, Margarita L. Alexandrova ${ }^{1}$, Irena I. Gencheva ${ }^{2}$}

Department of Neurology, University Hospital - Pleven ${ }^{1}$ Department of Biophysics, Medical University - Pleven ${ }^{2}$ Medical Diagnostic Clinical Laboratory, University Hospital - Pleven, Bulgaria

\begin{abstract}
Summary
Individuals with hypertension and diabetes mellitus are at high risk of cerebrovascular and cardiovascular morbidity and mortality. Recent advances in the multifactorial pathophysiology of atherogenesis provide important information about the complex interrelations between traditional risk factors, inflammation and oxidative stress in mediating all stages of atherosclerosis. The objective of the study was to determine if some inflammatory and oxidative stress markers in patients with arterial hypertension and diabetes mellitus differ from those in healthy age-matched controls. Our results revealed a significant difference in blood pro/antioxidant activities in hypertensive diabetics and the controls. The investigation of inflammatory and oxidative stress markers along with traditional risk factors proves useful in complex assessment of vascular risk and primary prophylaxis of cerebrovascular and cardiovascular events.
\end{abstract}

Key words: diabetes, hypertension, inflammation, oxidative stress, C-reactive protein

\section{Introduction}

Arterial hypertension and diabetes mellitus play a crucial role in the pathogenesis of atherosclerosis and atherosclerotic complications. The combination of arterial hypertension and diabetes mellitus accounts for a large proportion of cardiovascular and cerebrovascular morbidity and mortality. Recent investigations on the role of inflammation in mediating all stages of atherosclerosis provide important information about the complex interrelations between traditional vascular risk factors and atherogenesis $[1,2]$. Persistent low-grade inflammation indicated by the levels of inflammatory markers predicts future coronary or cerebrovascular events, especially in patients with arterial hypertension and diabetes mellitus, and both conditions are recognized as pro-inflammatory conditions [3-7]. There is increasing experimental evidence that high-sensitivity C-reactive protein (hsCPR) is a powerful and reliable marker for assessment of vascular risk. However, it is still not routinely measured in clinical practice [8]. During the last decades several studies have examined the 
potential role of oxidative stress in atherogenesis. Evidence suggests that the most important risk factors for atherosclerosis like arterial hypertension and diabetes mellitus increase the production of free reactive oxygen species (ROS) [9]. Although literary data exist to support the correlation between increased oxidative stress and various vascular diseases, the findings for antioxidant enzymes activity and the effects of antioxidant administration for prevention of coronary and cerebral events are quite controversial [10]. The complex interrelation between arterial hypertension, diabetes mellitus, oxidative stress and antioxidant defense need further clarification.

The objective of the present study was to explore some inflammatory and oxidative stress markers and to determine if they are different in hypertensive diabetics and healthy age-matched controls.

\section{Patients and Methods}

We studied 19 diabetic hypertensive individuals (8 males and 11 females), median age 63 (59-67) years and 18 age-matched healthy controls (7 males and 11 females). Patients with acute or chronic infection, coexisting liver, kidney or cancer diseases and those who underwent surgery were not included in the study. Fasting venous blood was collected and total cholesterol, triglycerides, glucose level and blood cell count were measured using standard laboratory methods. Concentrations of hs-CRP were measured by immunoturbidimetric assay on Cobas Integra 400 with CRP LT 300T (Roche Diagnostic). Levels of serum lipid hydroperoxides (ROOH), activity of CopperZink Superoxid Dismutase (CuZn SOD), Catalase (CTS) and Glutathione Peroxidase (GSH-Px), Sulfhydryl (SH)-groups and Total Antioxidant State (TAS) were also measured.

All experiments were conducted in accordance with the rules and regulations approved by the University Research Ethics Committee. Informed consent was obtained from all the subjects studied. The statistical analysis was performed with the Statistical Package of Social Sciences 19.0 (SPSS Inc., Chicago, IL). Normality of distribution was checked with the Shapiro-Wilk test. The interval variables were represented as mean $( \pm$ standard deviation, SD) or median (25th-75th percentile) depending on the type of distribution. The significance of differences between the groups was assessed by one-way ANOVA for normally distributed data along with the Kruskal-Wallis test for nonparametric data. As data were not normally distributed, the significance of differences between the patient groups was assessed with the Mann-Whitney test. A value of $\mathrm{p}<0.05$ was considered statistically significant.

\section{Results}

The baseline characteristics of hypertensive diabetic patients and healthy age-matched controls included in the present study are given in Table 1.

We found increased white blood cell count $(\mathrm{Z}=-1.963, \mathrm{p}=0.049)$ (Figure 1) and platelets $(\mathrm{F}=11.979, \mathrm{p}=0.001)$ and significantly higher levels of hs-CRP $(Z=-3.723, p=0.000)$ (Figure 2) in patients with arterial hypertension and diabetes mellitus.

Triglycerides were increased in the blood of hypertensive diabetics $(\mathrm{Z}=-3.237, \mathrm{p}=0.001)$. There was no significant difference between total cholesterol levels found in the hypertensive diabetics and the controls.

Increased activity of CuZn SOD ( $\mathrm{F}=4.399$, $\mathrm{p}=0.043)$, decreased activity of GSH-Px $(\mathrm{Z}=-$ $3.006, p=0.003)$ and decreased level of $\mathrm{SH}-$ groups $(Z=-3.249, p=0.00)$ were found in hypertensive diabetics. Blood CTS activity and serum levels of TAS remained unchanged in hypertensive diabetics. The serum level of $\mathrm{ROOH}$ was significantly higher in patients with arterial hypertension and diabetes mellitus as compared with the level in the control group $(\mathrm{F}=9.739, \mathrm{p}=0.004)$.

\section{Discussion}

Inflammation plays a fundamental role in atherogenesis [5]. Evidence from experimental and clinical studies implicates inflammatory mechanisms in the initiation and progression of atherosclerosis [4]. Furthermore, data exists that some inflammatory parameters could even modify the risk of atherosclerotic complications regardless of the presence of traditional risk factors [11]. According to Saito et al. [12] markers of inflammation such as CRP and leukocyte count are not only associated with an increased risk of atherosclerotic complications but might predict future myocardial infarction or stroke. At present, the complex interrelations 
Table 1. Baseline characteristics of patients.

\begin{tabular}{|c|c|c|}
\hline Parameter & $\begin{array}{l}\text { Control group } \\
n=18\end{array}$ & $\begin{array}{l}\text { Patients } \\
n=19\end{array}$ \\
\hline Age [years] & $57 \pm 9$ & $61 \pm 9$ \\
\hline Male sex, n (\%) & $7(39)$ & $8(45)$ \\
\hline \multicolumn{3}{|l|}{ Vascular risk factors } \\
\hline $\mathrm{SBP}[\mathrm{mm} \mathrm{Hg}]$ & $125 \pm 12.6$ & $155 \pm 13^{\#}$ \\
\hline DBP [mm Hg] & $79 \pm 6.2$ & $90 \pm 4.7^{\#}$ \\
\hline Diabetes mellitus, n (\%) & $0(0)$ & $19(100)$ \\
\hline Alcohol abuse, n (\%) & $0(0)$ & $2(11)$ \\
\hline Cigarette smoking, n (\%) & $0(0)$ & $2(11)$ \\
\hline \multicolumn{3}{|l|}{ Laboratory parameters } \\
\hline $\mathrm{WBC}\left[\times 10^{9} / 1\right]$ & $6.7 \pm 2.5$ & $7.4 \pm 2.4^{\#}$ \\
\hline PLT $\left[\times 10^{9} / 1\right]$ & $244.5 \pm 37.3$ & $288 \pm 66^{\#}$ \\
\hline $\mathrm{RBC}\left[\mathrm{x} 10^{12} / 1\right]$ & $4.7 \pm 0.5$ & $4.3 \pm 0.4$ \\
\hline GLU $[\mathrm{mmol} / 1]$ & $\begin{array}{l}5.5 \pm 0.5 \\
5.5(5.1-5.7) \\
\end{array}$ & $\begin{array}{l}9.8 \pm 3.9^{\#} \\
8.2(6.9-13.1) \\
\end{array}$ \\
\hline $\mathrm{CHOL}[\mathrm{mmol} / \mathrm{l}]$ & $5.8 \pm 0.6$ & $5.7 \pm 1.4$ \\
\hline Fibrinogen $[\mathrm{g} / \mathrm{l}]$ & $\begin{array}{l}3.1 \pm 0.7 \\
3(2.7-3.2)\end{array}$ & $\begin{array}{l}3.0 \pm 0.5 \\
2.9(2.8-3.1)\end{array}$ \\
\hline TG $[\mathrm{mmol} / 1]$ & $\begin{array}{l}1.2 \pm 0.3 \\
1.1(0.9-1.4)\end{array}$ & $\begin{array}{l}2.2 \pm 1.1^{\#} \\
1.7(1.3-3.4) \\
\end{array}$ \\
\hline TAS [mmol/Trolox/l] & $\begin{array}{l}1.3 \pm 0.3 \\
1.2(0.9-1.3) \\
\end{array}$ & $\begin{array}{l}1.3 \pm 0.4 \\
1.2(1.1-2.9) \\
\end{array}$ \\
\hline hs-CRP [mg/l] & $\begin{array}{l}1.5 \pm 0.2 \\
0.9(0.6-1.3)\end{array}$ & $\begin{array}{l}6.8 \pm 2.0^{\#} \\
3.2(1.6-7.5)\end{array}$ \\
\hline $\mathrm{ROOH}[\mathrm{nmol} / \mathrm{ml}]$ & $3.6 \pm 0.6$ & $4.6 \pm 1.6^{\#}$ \\
\hline $\mathrm{SH}[\mu \mathrm{mol} / \mathrm{ml}$ кръв] & $4.5 \pm 0.5$ & $3.8 \pm 0.5^{\#}$ \\
\hline GSH-Px $[\mu \mathrm{mol} / \mathrm{GSSG} / \mathrm{min} / \mathrm{ml}]$ & $10.2 \pm 1.2$ & $9.0 \pm 0.9^{\#}$ \\
\hline $\mathrm{CuZn} \mathrm{SOD}\left[\mathrm{U} / 10^{9} \mathrm{RBC}\right]$ & $37.9 \pm 11.0$ & $47.6 \pm 14.8^{\#}$ \\
\hline
\end{tabular}

Data are presented as mean value $\pm \mathrm{SD}$ or mediana (25-75 percentile); ${ }^{\#} \mathrm{p}<0.05$ - significant difference between the patients and the control group

between inflammatory markers and some vascular risk factors like arterial hypertension and diabetes are not completely understood, and the sources of persistent low grade inflammation are not well defined [13]. For example, Angiotensin II could be in part responsible for mediating vascular inflammation, triggering oxidative stress, up-regulation of proinflammatory transcription factors that regulate the generation of inflammatory mediators and lead to endothelial dysfunction and vascular damage [14]. Recently, the so called acute phase reactants have been studied as potential markers of persisting or ongoing subclinical systemic vascular alterations [3]. Our findings suggest the existence of increased immune status in diabetic hypertensive individuals, reflected by enhanced white blood cell count $(p=0.049)$ and significantly higher serum hs-CRP levels $(p=0,000)$ as compared with the control subjects.

However, it is still unknown whether these raised inflammatory parameters are only markers of low grade inflammation or active contributors to development and progression of atherosclerotic complications [15]. It is proposed that increased levels of inflammatory markers, especially in diabetic hypertensive individuals, are associated with ongoing vascular pathology and could predict increased risk of vascular events $[13,14]$. Data have also been published 


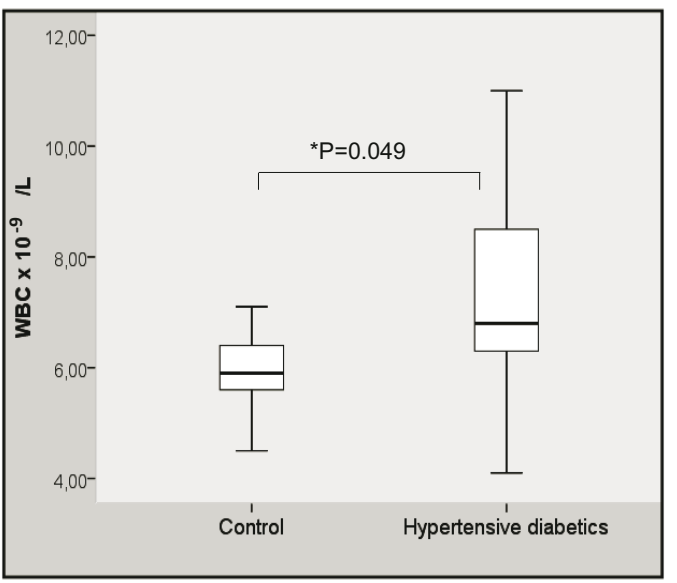

Figure 1. White blood cell count of hypertensive diabetics and control subjects

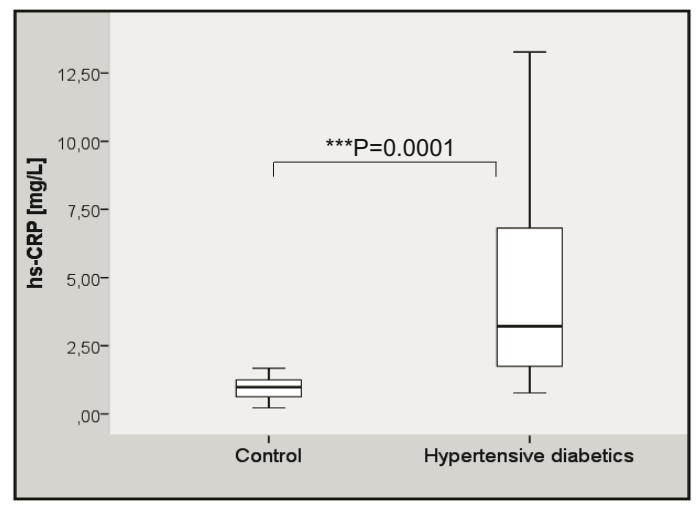

Figure 2. Serum hs-CRP in hypertensive diabetics and control subjects

that the acute phase reactant CRP is directly involved in the progression of vascular damage by connecting and activating complement, inducing adhesion molecules expression, mediating LDL capture by endothelial macrophages and inducing accumulation of monocytes in the arterial wall $[15,16]$.

Dyslipidemia is considered one of the major etiologic factors for atherosclerosis and is often present in patients with arterial hypertension and diabetes mellitus [17]. Our results revealed increased level of triglycerides, while total cholesterol did not differ in hypertensive diabetics versus the control subjects. Such a finding is in contradiction with the literary data published [10]. A prolonged treatment with statins is the most acceptable explanation of dyslipidemia found in the patients with arterial hypertension and diabetes mellitus.

Oxidative stress is a condition in which 92 generation of ROS exceeds the capacity of the antioxidant defense system. Persistent oxidative stress can deplete antioxidant molecules, inactivate antioxidant enzymes, thereby impairing the antioxidant defense system. Furthermore, persistent pro/antioxidant imbalance leads to endothelial dysfunction, an initial step of atherogenesis. There is compelling evidence that oxidative stress plays a critical part in the pathogenesis of arterial hypertension. It also participates in the structural and functional alterations in the cerebral blood vessels. The central role of oxidative stress in the atherosclerotic process has been studied in numerous epidemiological and experimental studies but the complex interrelations between oxidative stress, on the one hand, and the pathophysiology of arterial hypertension and diabetes mellitus, on the other, have not been completely defined. Data published on the activity of some antioxidant enzymes are insufficient and contradictory [10]. Increased activity of erythrocyte CuZnSOD in patients with arterial hypertension and diabetes mellitus was found in the present study. A compensatory reaction to overproduction of superoxide in the circulation, induced by arterial hypertension and diabetes mellitus was probably responsible for the increased activity of CuZnSOD. CTS activity of hypertensive diabetics was unchanged, but GSH-Px and the level of SH-groups were lower in the blood of hypertensive diabetics. Reduction of the antioxidant capacity following an overproduction of ROS is the most acceptable reason of such study results.

Patients with arterial hypertension had lower activity of antioxidant enzymes, as compared to the healthy controls. A decreased level of GSHPx was found in patients with diabetes $[9,18,19]$. No significant difference concerning TAS in hypertensive diabetics was established probably due to increased uric acid in the blood of hypertensive diabetics [18].

Oxidative stress is associated with increased lipid peroxidation, followed by elevation of serum lipid hydroperoxides. Few studies have reported increased levels of malondialdehyde (MDA), correlated with the history of diabetes mellitus, regardless of gender $[10,18]$. Increased MDA has also been registered in patients with arterial hypertension without diabetes mellitus [17]. Different pathophysiological mechanisms are implicated in the oxidative damage, but hyperlipidemia is mostly responsible for increased lipid peroxidation in hypertensive diabetics [17]. The higher level of serum $\mathrm{ROOH}$ 
in hypertensive diabetics found probably reflects the pathogenesis and progression of arterial hypertension and diabetes mellitus.

\section{Conclusion}

In conclusion, our results demonstrate the presence of increased immune state and oxidative stress in hypertensive diabetic patients versus control subjects, probably associated with the progression of atherosclerosis and its complications like stroke and myocardial infarction. Future prospective large populationbased studies could improve basic knowledge on the complex interrelations between vascular risk

\section{References}

1. Libby P, Ridker P, Maseri A. Inflammation and atherosclerosis. Circulation. 2002;105(9):113543.

2. Lind L. Circulating markers of inflammation and atherosclerosis. Atherosclerosis. 2003;169(2): 203-14.

3. Danesh J, Whincup P, Walker M, Lennon L, Thomson A, Appleby $\mathrm{P}$, et al. Low grade inflammation and coronary heart disease: prospective study and updated meta-analyses. BMJ. 2000;321(7255):199-204.

4. Castoldi G, Galimbertim S, Riva C, Papagna R, Querci F, Casati M, et al. Association between serum values of $\mathrm{C}$-reactive protein and cytokine production in whole blood of patients with type 2 diabetes. Clin Sci(Lond). 2007;113(2):103-8.

5. Elkind M. Inflammatory mechanisms of stroke. Stroke. 2010; 41(10 Suppl):S3-S8.

6. Libby P, Ridker P. Inflammation and atherosclerosis: role of $\mathrm{C}$-reactive protein in risk assessment. Am J Med. 2004;116(6 Suppl 1):9S$16 \mathrm{~S}$.

7. Ross R. Atherosclerosis: an inflammatory disease. NEngl J Med. 1999;340(2):115-26.

8. Di Napoli M, Elkind M, Godoy D, Singh P, Papa F, Popa-Wagner A. Role of C-reactive protein in cerebrovascular disease: a critical review. Expert Rev Cardiovasc Ther. 2011;9(12):1565-84.

9. Pasupathi P, Farook J, Chinnaswamy P. Oxidantantioxidant status, high sensitive C-reactive protein and homocysteine levels in type 2 diabetic patients with and without microalbuminuria. Int $\mathrm{J}$ Biol Med Res. 2010;1(3):04-8.

10. Likidlilid A, Patchanans N, Peerapatdit T. Lipid peroxidation and antioxidant enzyme activities in erythrocytes of type 2 diabetic patients. J Med Assoc Thai. 2010;93(6):682-93. factors, low-grade inflammation and oxidative stress responsible for progressive vascular damage. New scientific achievements will answer the question how some indicators of increased immune state and oxidative stress could serve as additional markers for prediction of cardiovascular and cerebrovascular risk. Furthermore, a potential involvement of inflammatory and oxidative stress markers in the pathogenesis of vascular complications calls for debate over the use of anti-inflammatory and antioxidant treatment in the complex therapeutic approach for primary prophylaxis of future coronary and cerebral vascular events in highrisk diabetics with hypertension.

11. Lindsberg P, Grau A. Inflammation and infections as risk factors for ischemic stroke. Stroke. 2003;34(10):2518-32.

12. Saito M, Ishimittsu T, Minami J, Ono H, Ohrui M, Matsuoka H. Relations of plasma high-sensitivity C-reactive protein to traditional risk factors. Atherosclerosis. 2003;167(1):73-9.

13. Anand A, Manikandan R, Begum T, Kumar K, Akilandeswari G, Jerline M, et al. Hypertension, diabetes, metabolic syndrome, inflammation and the risk of stroke. Int $\mathrm{J}$ Biol Med Res. 2011;2(1):429-32.

14. Savoia C, Schiffrin E. Vascular inflammation in hypertension and diabetes: molecular mechanisms and therapeutic interventions. Clin Sci (Lond). 2007;112(7):375-84.

15. Di Napoli M, Papa F. Inflammation, hemostatic factors and antithrombotic agents in relation to long-term risk of new cardiovascular events in first-ever ischemic stroke patients. Stroke. 2002; 33:1763-71.

16. Ridker PM, Silvertown JD. Inflammation, Creactive protein, and atherothrombosis. J Periodontol. 2008;79(8 Suppl):1544-51.

17. Biswas UK, Kumar A. Study on lipid profile, oxidation stress and carbonic anhydrase activity in patients with essential hypertension. J Clin Diag Res. 2010;4:3414-20.

18. Kusano C, Ferrari B. Total antioxidant capacity: a biomarker in biomedical and nutritional studies. $\mathrm{J}$ Cell Mol Biol. 2008;7(1):1-15.

19. Mahboob M, Rahman M, Grover P. Serum lipid peroxidation and antioxidant enzyme levels in male and female diabetic patients. Singapore Med J. 2005;46(7):322-4. 